INRA Prod. Anim., $2005,18(4), 231-240$

\section{Combiner la gestion optimale du pâturage et les performances des vaches laitières : enjeux et outils}

\author{
J.-L. PEYRAUD, L. DELABY
}

INRA, UMR Production du Lait, F-35590 Saint-Gilles

Courriel :jean-louis.peyraud@rennes.inra.fr

Les travaux de recherches récents sur l'alimentation des vaches laitières au pâturage et la gestion des surfaces pâturées ont permis le développement de recommandations pratiques et d'outils d'aide à la décision qui devraient permettre aux éleveurs de mieux valoriser cette ressource fourragère.

Depuis l'instauration de la PAC, la pratique d'un prix du lait élevé a encouragé les systèmes de production laitière à haut niveau d'intrants (fertilisation, concentrés, fourrages stockés) au détriment du pâturage. Cette évolution a été renforcée par la simplicité d'utilisation de l'ensilage de maïs chez des troupeaux conduits en vêlages d'automne et par l'incapacité du pâturage à maximiser les performances laitières individuelles sans cesse croissantes. Ainsi, le rôle du pâturage dans l'alimentation des vaches laitières a diminué durant ces 30 dernières années (Bourgeois 2002). Tout en maintenant le régime des quotas laitiers, la récente réforme de la politique laitière européenne va supprimer la protection tarifaire appliquée sur le beurre et la poudre de lait (Chausson 2004). Le prix du lait payé aux producteurs risque alors de baisser plus que le montant de l'aide compensatrice accordée lors de la réforme de Luxembourg. Ces événements renforcent la nécessité d'une meilleure efficacité (exprimée ici par tonne de quota à produire) des systèmes de production laitière. Dans le même temps, des efforts importants devront être réalisés afin de réduire l'impact négatif sur l'environnement et les ressources naturelles, conséquence de pratiques agronomiques inappropriées (Le Gall et al 2004). La nouvelle PAC et l'introduction de mesures agro-environnementales plus strictes risquent de générer de nouvelles priorités en matière d'utilisation du territoire et d'environnement avec des répercussions sur l'utilisation des intrants.

Dans le futur, les prairies et le pâturage devraient constituer le socle des sys- tèmes de production laitière durables puisque l'herbe pâturée reste l'aliment le moins onéreux pour nourrir les vaches laitières. Le pâturage peut aussi contribuer à limiter les risques environnementaux liés aux systèmes les plus intensifs, tout en favorisant les effets favorables de l'activité d'élevage dans le maintien ou la gestion de la biodiversité et des paysages (Parris 2002, Chatellier et Vérité 2003). D'autre part, le pâturage constitue un élément de réponse à la demande croissante des consommateurs favorables à un retour à des méthodes de production plus «naturelles», perçues comme plus sécurisantes et qui renforcent le lien entre produit et terroir (Bourgeois 2002).

Une utilisation efficace de l'herbe par le pâturage associée à un usage limité des concentrés et de la fertilisation azotée requiert la mise au point de systèmes maximisant l'ingestion d'herbe tout en assurant la pérennité du système en terme de quantité et de qualité de l'herbe produite. Cela nécessite aussi le développement d'outils d'aide à la décision d'utilisation simple qui doivent permettre à l'éleveur d'organiser dans le temps et l'espace l'utilisation de cette ressource fourragère instable. De tels outils devraient redonner confiance aux éleveurs dans le pâturage et ainsi permettre d'accroître la part de l'herbe pâturée dans l'alimentation annuelle des vaches laitières.

L'objectif de ce texte est de définir les conditions favorables au développement de systèmes herbagers efficaces et de souligner les marges de progrès possibles. Plus précisément, ce texte s'attachera à i) préciser les possibilités d'ali- menter des vaches laitières à haut potentiel au pâturage, ii) analyser les effets des pratiques de pâturage sur les performances individuelles et par hectare à l'échelle de la saison de pâturage, iii) recenser les nouveaux outils et méthodes facilitant la prise de décision à l'échelle de la parcelle et plus globalement du système de pâturage.

\section{1 / La vache et l'herbe ou comment accroître les quantités d'herbe ingérées et les performances indivi- duelles au pâturage}

\section{1 / Les quantités d'herbe ingé- rées augmentent avec les besoins des animaux}

Les vaches à haut niveau de production ont des besoins en nutriments plus élevés, qui se traduisent au pâturage par un accroissement des quantités d'herbe ingérées. Afin de prévoir l'ingestion d'herbe au pâturage, Peyraud et al (1996) ont proposé d'utiliser la production de lait attendue, compte tenu du potentiel des animaux et de leur stade de lactation, plutôt que la production de lait observée (PLobs). Le lait attendu (PLatt) peut être calculé à partir du lait au pic (PLmax) en intégrant une persistance hebdomadaire de 0,985 et le temps écoulé entre le lait au pic (admis en $6^{\mathrm{e}}$ semaine de lactation) et la période de pâturage considérée $($ PLatt $=$ PLmax * 0.985 (stade - 6) avec stade exprimé en semaine de lactation, Delaby et al 2003). Les travaux de Peyraud et al (1996) et de Delagarde et al (2001b), 
réalisés en mai et juin avec des vaches vêlant en automne, ont permis de montrer que l'ingestion d'herbe augmente d'environ $180 \mathrm{~g}$ de MS par kg de lait au pic ou de $250 \mathrm{~g}$ de MS par kg de lait attendu. Les données récentes de Kennedy et al (2002a) confirment les résultats précédents avec une augmenpic chez des vaches au $4^{\mathrm{e}}$ mois de lactation. Cette valeur chute à $120 \mathrm{~g}$ de MS au $8^{\mathrm{e}}$ mois de lactation. La pente de la relation obtenue en utilisant la production laitière observée (PLobs) durant les mesures d'ingestion est beaucoup plus élevée (400 à $500 \mathrm{~g}$ de MS, Stakelum et Connolly 1987, Peyraud non publié), ce qui traduit bien le caractère limitant de l'ingestion dans les performances des vaches laitières au pâturage.

Comme pour l'ingestion, le potentiel des animaux joue un rôle déterminant sur la production laitière des vaches au pâturage. A partir d'une série d'expériences incluant 236 vaches, Delaby et al (2001) et Delaby et Peyraud (2003) ont montré que la production laitière observée au printemps (d'avril à juin) est très étroitement reliée à la production laitière attendue (figure 1). La relation obtenue est la suivante :$$
\text { avec } \mathrm{n}=236-\text { Syx }=2,17-\mathrm{R}^{2}=0,74 \text { (1) }
$$

Selon cette équation, les vaches sont capables de produire avec de l'herbe seule, environ $60 \%$ de chaque kilo de lait attendu au dessus de $15 \mathrm{~kg}$. La pente de cette relation est cohérente avec celle décrite pour l'ingestion (250 g de MS par kg de PLatt) qui cortation de $190 \mathrm{~g}$ de MS par kg de lait au

$$
\text { PLobs }=6,3+0,59 \times \text { PLatt }
$$

Figure 1. Relation entre la production laitière attendue et observée durant le printemps chez des vaches laitières au pâturage sans apport de concentré.

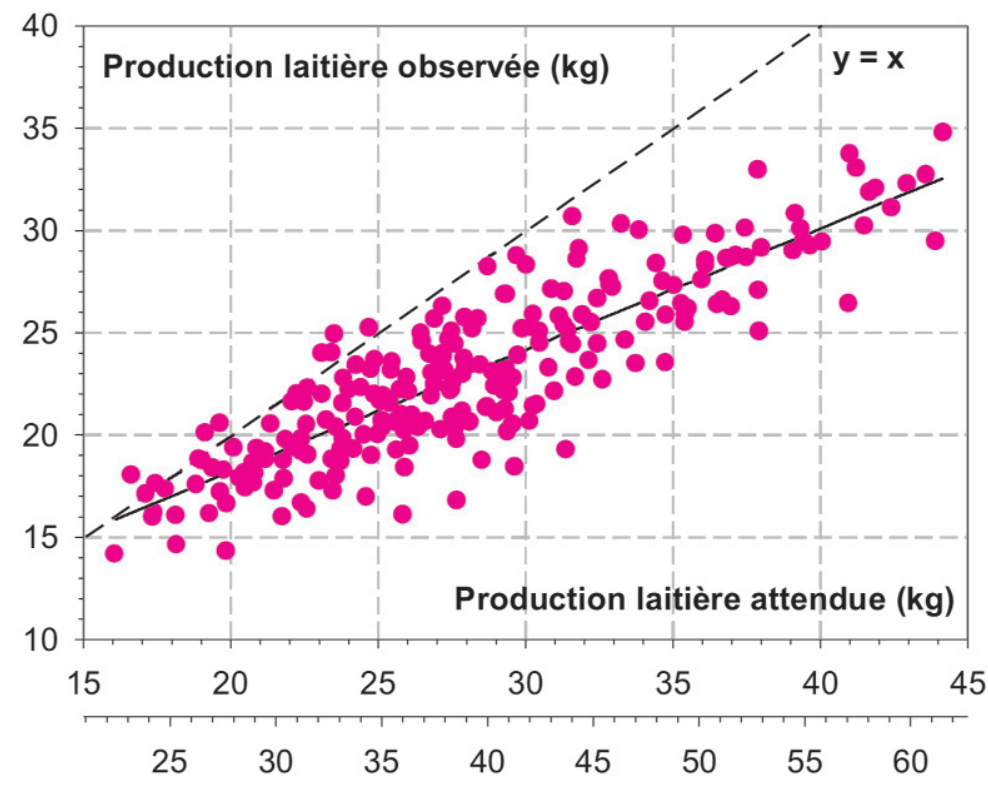

Lait au pic de lactation ( $k g$ - vêlage en novembre) respond à la couverture de $2 / 3$ environ des besoins énergétiques supplémentaires associés à chaque $\mathrm{kg}$ de PLatt. Cet effet du potentiel des animaux sur leurs performances au pâturage reste valable durant toute la saison de pâturage.

Ainsi, en système herbager, la production de vaches à fort index génétique est supérieure de 2 à $3 \mathrm{~kg}$ de lait par jour à celles de moindre potentiel, soit environ 800 à $1200 \mathrm{~kg}$ de lait en plus par vache et par an (Mc Gilloway et Mayne 1996, Buckley et al 2000, Kennedy et al 2002b). L'équation 1 confirme également que l'utilisation de l'herbe pâturée comme seul aliment de la ration ne per-
Figure 2. Influence des quantités d'herbe offertes journalières au ras du sol sur les quantités d'herbe ingérées par les vaches laitières et la valorisation de l'herbe en pâturage tournant.

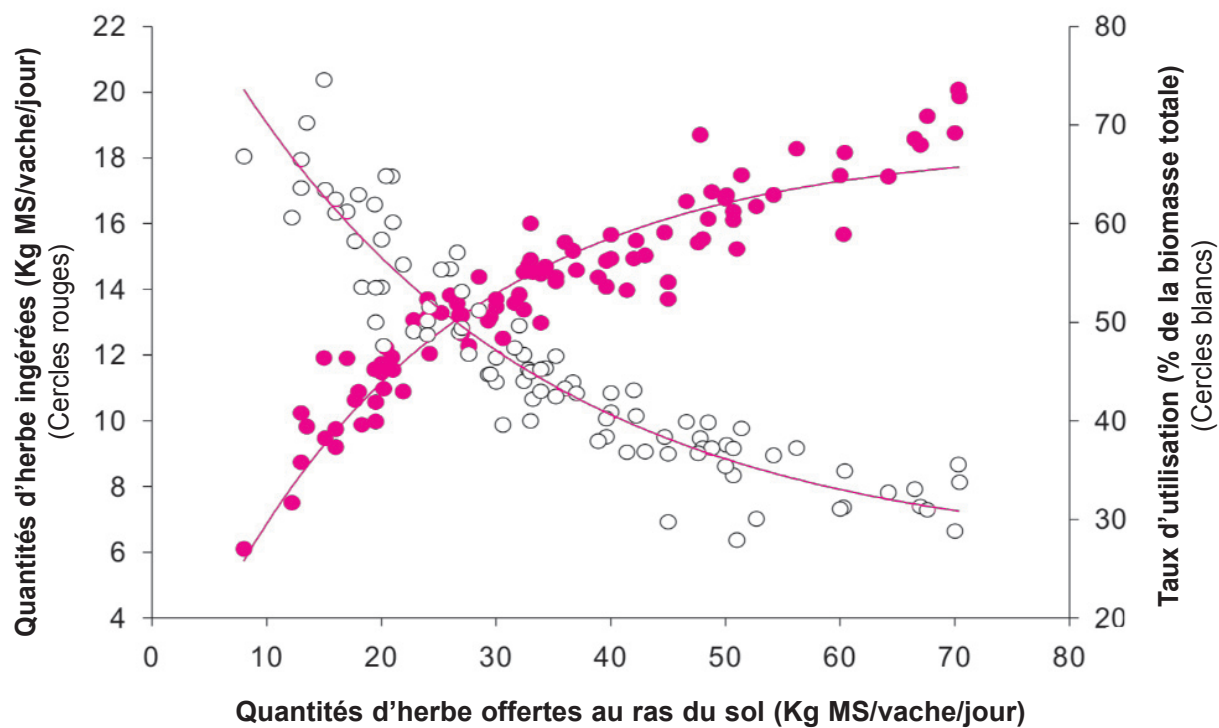

met pas aux vaches à haut potentiel de l'exprimer pleinement. L'écart entre le lait attendu et le lait effectivement produit est d'autant plus important que l'animal a un potentiel élevé. Cependant, le niveau de production moyen de $7400 \mathrm{~kg}$ de lait obtenu avec seulement $320 \mathrm{~kg}$ de concentré dans les travaux de Kennedy et al (2002b) montrent bien l'aptitude de ce type de vaches en système herbager, à condition toutefois de leur assurer des quantités d'herbe offertes élevées tout au long de la saison de pâturage.

\section{2 / Accroître les quantités d'herbe offertes pour accroître les quantités d'herbe ingérées}

En système de pâturage tournant, les conditions de pâturage sont souvent décrites à travers la notion de quantité d'herbe offerte $(\mathrm{QHO}$, exprimée en $\mathrm{kg}$ $\mathrm{MS} /$ vache/jour), qui correspond à la biomasse présente au dessus d'une certaine hauteur d'herbe. Le plus souvent, cette hauteur est fixée au ras du sol ou à 4 ou $5 \mathrm{~cm}$, admettant alors que l'herbe présente sous cette hauteur n'est pas disponible pour l'animal. A partir d'une revue bibliographique quantitative intégrant surtout des essais de courte durée, Delagarde et al $(2001 \mathrm{~b}, 2004 \mathrm{a}-$ figure 2) ont obtenu une solide relation curvilinéaire entre quantités d'herbe offertes mesurées au ras du sol et quantités d'herbe ingérées (QHI en $\mathrm{kg}$ $\mathrm{MS} /$ vache/jour) :

$$
\begin{gathered}
\mathrm{QHI}=18,4 *\left(1-\mathrm{e}^{-0,0466 * \mathrm{QHO}}\right) \\
\text { avec } \mathrm{n}=92-\mathrm{Syx}=0,99-\mathrm{R}^{2}=0,87
\end{gathered}
$$


Selon cette relation, l'ingestion d'herbe n'augmente plus pour des quantités d'herbe offertes supérieures à $60 \mathrm{~kg}$ MS/jour. Avec un tel niveau d'offert, les vaches sont capables de satisfaire leur appétit. Pour des quantités d'herbe offertes classiquement rencontrées en Europe et comprises entre 25 et $40 \mathrm{~kg} \mathrm{MS} /$ jour (calculées au ras du sol), l'ingestion des vaches laitières reste inférieure à $80 \%$ de leur capacité d'ingestion. Cette limitation de l'ingestion permet de comprendre pourquoi ces animaux ne sont pas capables d'exprimer pleinement leur potentiel avec de l'herbe seule. Dans cette gamme de quantités offertes, les quantités ingérées augmentent de $150 \mathrm{~g}$ par $\mathrm{kg}$ de QHO exprimé au ras du sol et de 200 à $250 \mathrm{~g}$ par $\mathrm{kg}$ de $\mathrm{QHO}$ exprimé à $5 \mathrm{~cm}$.

Comme la hauteur d'herbe en sortie de parcelle est une fonction directe des quantités d'herbe offertes, un accroissement d'ingestion de $1 \mathrm{~kg}$ de MS/ vache/jour, obtenue par augmentation des quantités offertes de $4 \mathrm{~kg}$ de MS, se traduit par une augmentation d'environ $0,7 \mathrm{~cm}$ de la hauteur d'herbe en fin de parcelle, mesurée avec un herbomètre à plateau. Dans ces conditions, l'ingestion supplémentaire d'herbe ne représente alors que le quart des quantités d'herbe offertes en plus. Ce résultat explique l'augmentation rapide de la hauteur en sortie de parcelle avec l'accroissement des quantités d'herbe offertes. En conséquence, une conduite libérale du pâturage au printemps en vue d'accroître les quantités d'herbe offertes et/ou les performances des vaches laitières se traduit par une dégradation de la qualité de l'herbe en milieu et fin de saison et une baisse des per-formances lors des cycles successifs de pâturage (Mayne et al 1987, Hoogendoorn et al 1992). Les possibilités d'accroître l'ingestion par les quantités d'herbe offertes s'avèrent donc limitées sur le long terme et des stratégies alternatives doivent être imaginées pour augmenter durablement ingestion et production laitière au pâturage.

\section{3 / Accroître la disponibilité de l'herbe et sa qualité pour accrô̂- tre les quantités d'herbe ingé- rées}

La disponibilité de l'herbe peut être définie comme la facilité avec laquelle l'herbe est récoltée par l'animal au pâturage. Il s'agit d'un paramètre complexe qui prend en compte à la fois des aspects quantitatifs et qualitatifs du couvert végétal (hauteur, \% de limbes) et les interactions avec les quantités d'herbe offertes. En système de pâturage continu, les quantités d'herbe offertes sont théoriquement illimitées et l'ingestion s'accroît selon une loi asymptotique avec la biomasse par hectare et/ou la hauteur du couvert végétal. La synthèse bibliographique réalisée par Delagarde et al (2001b) a permis de quantifier la relation entre les quantités d'herbe ingérées et la hauteur du couvert mesurée au «sward stick»: cette relation, établie pour une vache de $600 \mathrm{~kg}$ de poids vif, a pour équation :

$$
\begin{gathered}
\mathrm{QHI}=17,6 *\left(1-\mathrm{e}^{-0,303 * \mathrm{Hc}}\right) \\
\text { avec } \mathrm{n}=29-\mathrm{Syx}=1,43-\mathrm{R}^{2}=0,73
\end{gathered}
$$

La relation indique que l'ingestion maximale est obtenue pour une hauteur de $9-10 \mathrm{~cm}$ et qu'elle décroît rapidement pour des hauteurs inférieures à $7 \mathrm{~cm}$.

En pâturage tournant, la hauteur de l'herbe en début de parcelle est plus haute qu'en pâturage continu et elle diminue durant le temps de séjour des animaux en fonction des quantités d'herbe offertes. La disponibilité de l'herbe peut alors être en partie décrite par la proportion de feuille contenue dans la strate pâturée. Wade (1989, 1995) a été le premier à montrer que la disponibilité de l'herbe augmente avec la proportion de feuilles vertes restant dans le bas de la plante lorsque l'animal cesse de consommer. Ce résultat a ensuite été confirmé par Parga et al (2000) et Delagarde et al (2004b). Finalement, les quantités d'herbe ingérées au pâturage seraient mieux prédites à partir des quantités de feuilles vertes offertes (Syx $=1,46 \mathrm{~kg}$ DM) qu'avec les quantités de matière sèche offertes $($ Syx $=2,02 \mathrm{~kg}$ DM -

Figure 3. Relation entre les quantités d'herbe ou de limbes offertes et les quantités ingérées au pâturage.

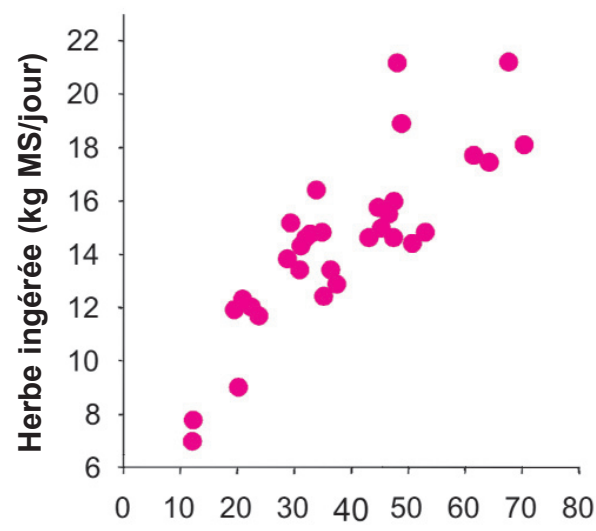

Quantité d'herbe offerte ras du sol (kg MS/jour) figure 3). Ainsi, l'augmentation de la biomasse de feuilles vertes dans le bas de la plante obtenue grâce à des pratiques de pâturage appropriées au début du printemps aura un rôle très favorable sur l'ingestion tout en conservant une faible biomasse résiduelle durant toute la saison de pâturage.

La composition botanique de la prairie peut également contribuer à accrô̂tre la disponibilité et la qualité de l'herbe pâturée. Ainsi, l'ingestion d'herbe et la production laitière sont plus élevées sur prairies d'association ray-grass anglais-trèfle blanc que sur prairies de ray-grass pur (Thomson et al 1985, Wilkins et al 1994 et 1995, Ribeiro-Filho et al 2003). La différence est d'autant plus importante que la proportion de trèfle dans l'association est élevée (Harris et al 1997). Les prairies d'association méritent une attention particulière dans la recherche d'alternatives aux graminées pures pour augmenter les performances des vaches laitières. Ce, d'autant plus, que l'introduction de légumineuses dans les prairies présente des avantages agronomiques et économiques intéressants associés à la réduction de fertilisation minérale azotée. A l'inverse, les performances des vaches laitières au pâturage sont peu affectées lors des comparaisons de variétés de ray-grass de même précocité (Hageman et al 1993, Gowen et al 2003). Il semble donc très difficile d'améliorer l'ingestion par la sélection de ray-grass anglais tout en gardant par ailleurs leurs caractéristiques agronomiques actuelles, telles que leur productivité et leur épiaison tardive. 


\section{2 / Les éleveurs et la gestion du pâturage ou comment améliorer la valorisation de l'herbe produite par le troupeau}

\section{1 / Accroître la période de croissance de l'herbe}

Dans les régions de l'Ouest de l'Europe (Grande Bretagne, Irlande, Bretagne, Normandie, ...), l'herbe pousse assez régulièrement du printemps à l'automne. Les régions les plus favorables caractérisées par des étés pluvieux ont un potentiel de production des prairies qui peut atteindre 15 tonnes de MS /ha ou encore $20000 \mathrm{~kg}$ de lait/ha (Holmes 1980). Dans d'autres régions (Pays de Loire, Sud de la France, Galice, ...), la courbe de croissance de l'herbe se caractérise par des valeurs très faibles à nulles en été qui obligent les éleveurs à rentrer les animaux en stabulation lorsque la surface en herbe disponible ne compense pas cette faible croissance (MosqueraLosada et Rodriguez 1999a). A l'opposé, l'arrêt de croissance sous l'effet des au Nord de l'Espagne que dans le Nord de l'Europe. Au sein même de régions réputées favorables telle que la Bretagne, il existe une variabilité pédoclimatique locale importante (pluviométrie estivale irrégulière ou limitée, sols à faible réserve utile) qui crée des disparités importantes dans le profil annuel de croissance de l'herbe (Defrance et al 2005 - figure 4). Au delà de ces effets associés aux saisons, tous les pays d'Europe connaissent des variations inter annuelles du profil de croissance de l'herbe. Finalement, il faibles températures intervient plus tard

Tableau 1. Intérêt d'un accès de durée limitée au pâturage en fin d'hiver sur les performances des vaches laitières en début de lactation.

\begin{tabular}{|l|c|c|c|c|}
\hline \multicolumn{1}{|c|}{ Auteurs } & \multicolumn{2}{|c|}{ Sayers et Mayne 2001 } & \multicolumn{2}{c|}{ Dillon et al 2002 } \\
\hline Alimentation (1) & $\begin{array}{c}\text { Ensilage } \\
\text { d'herbe }\end{array}$ & Pâturage (2 h) & $\begin{array}{c}\text { Ensilage } \\
\text { d'herbe }\end{array}$ & Pâturage (12 h) \\
\hline Ingestion (kg MS) & & $\begin{array}{c}\text { Apport d'ensilage } \\
\text { restreint }\end{array}$ & $\begin{array}{c}\text { Ensilage } \\
\text { à volonté }\end{array}$ \\
\hline Ensilage & 10,2 & 6,1 & 9,2 & 3,4 \\
Herbe pâturée & & 5,1 & & 9 \\
Lait (kg/vache/j) & 25,9 & 28,7 & 23,5 & 27,1 \\
TB (g/kg) & 39,9 & 40,5 & 36,1 & 38,2 \\
TP (g/kg) & 28,6 & 30,2 & 29,6 & 32 \\
\hline
\end{tabular}

(1) Apport de 5,5 kg de concentré. Résultats moyens obtenus au cours de 6 semaines d'expérience. reste un réel challenge à mieux maîtriser la croissance de l'herbe durant toute la saison de pâturage.

La productivité d'une prairie dépend beaucoup de la disponibilité en azote issue de la minéralisation et/ou de la fertilisation. L'apport d'azote minéral permet ainsi de moduler la croissance de l'herbe. En fin d'hiver, la date du premier apport d'azote joue un rôle important sur le démarrage de végétation et donc la date possible de mise à l'herbe (Lemaire et al 1981, O'Donovan et al 2004). Toutefois, l'influence de la fertilisation azotée varie selon les conditions climatiques. Par exemple, la réponse est plus importante dans les zones sous influence maritime qu'en altitude (Mosquera-Losada et Rodriguez 1999b). De même, l'apport d'azote en fin d'été stimule la croissance dès le retour des premières pluies à condition que la température et le rayonnement solaire ne deviennent pas des facteurs limitants.

Un objectif prioritaire des sélectionneurs en plantes fourragères reste $d$ 'ac-
Figure 4. Profil décadaire de croissance de l'herbe en Bretagne selon les conditions pédoclimatiques au cours du printemps, de l'été et l'automne.

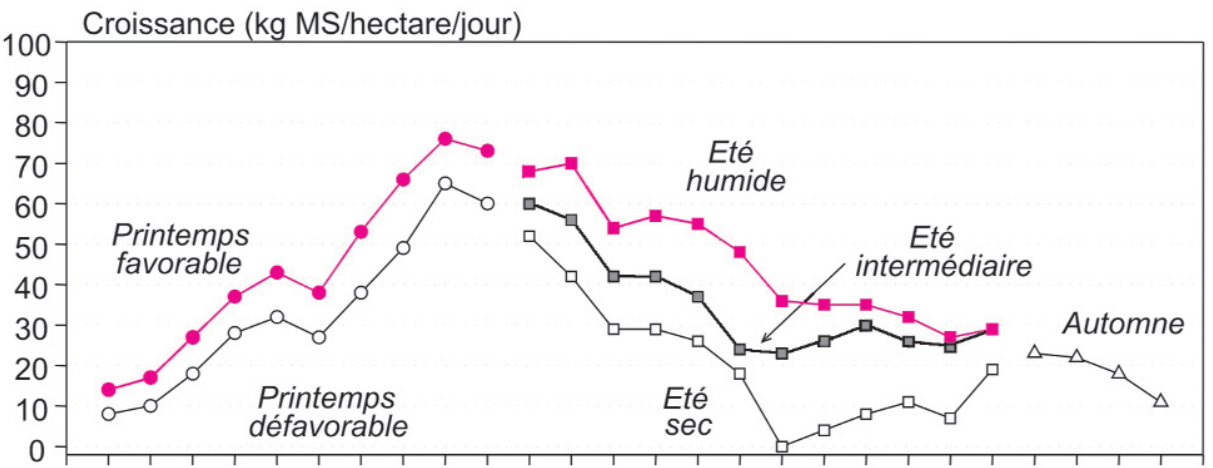

$\begin{array}{llllllllllllllllllllllllll}3 & 1 & 2 & 3 & 1 & 2 & 3 & 1 & 2 & 3 & 1 & 2 & 3 & 1 & 2 & 3 & 1 & 2 & 3 & 1 & 2 & 3 & 1 & 2 & 3 & 1\end{array}$

Fév Mars Avril Mai Juin Juill Août Sept Oct Nov

Source : Pâture Plus - GIS Agrotransfert Bretagne. croître la période de croissance des graservés sur les nouvelles variétés de ray-grass inscrites au catalogue proviennent pour l'essentiel d'une augmentation lente mais régulière de la durée de la saison de croissance et rien ne semble devoir limiter cette possibilité d'amélioration génétique (Wilson 1993). L'utilisation d'autres espèces comme le dactyle, la fétuque ou les associations avec légumineuses, réputées plus résistantes à la sécheresse et aux températures élevées que les raygrass doit permettre d'accroître la durée de la saison de pâturage. Bien que coûteuse et peu utilisée sur prairies, l'irrigation est aussi un moyen de limiter les déficits hydriques et donc de prolonger les phases de croissance de l'herbe en été.

\subsection{Accroître la durée de la sai-} son de pâturage : une opportunité pour consommer plus d'herbe et accroître les performances animales

Compte tenu de la valeur nutritive élevée de l'herbe en regard de celle des fourrages conservés, il parait opportun d'étendre la durée de la saison de pâturage. Bien que faible, la croissance de l'herbe en début de printemps ou celle d'automne qui est souvent perdue par sénescence durant l'hiver pourrait ainsi être mieux valorisée. Les expériences conduites à Hillsborough en Irlande du Nord (Sayers et Mayne 2001) et à Moorepark en République d'Irlande (Dillon et al 2002) ont bien montré qu'un accès de durée limitée au pâturage (3-4 heures/jour) en février-mars ou en novembre-décembre (Mayne et Laidlaw 1995) permettait d'améliorer la production laitière de 2 à $3 \mathrm{~kg}$ de lait par vache et par jour et de réduire la consommation d'ensilage d'herbe de 4 à $6 \mathrm{~kg} /$ jour (tableau 1). minées. Les gains de productivité ob- 
L'intérêt du pâturage d'automne en complément des rations à base d'ensilage de maïs a été confirmé en Bretagne (Chenais et Roux 1996). Les vaches ayant accès aux prairies durant la journée (6 heures/jour) ont produit $1 \mathrm{~kg}$ de lait en plus et consommé $5,1 \mathrm{~kg}$ d'ensilage de maïs en moins que les vaches en stabulation permanente. L'intérêt de ces pratiques de pâturage précoces ou tardives en saison réside notamment dans la réduction des coûts de production moins élevés avec l'herbe pâturée qu'avec les fourrages stockés. Pendant ces périodes, il faut néanmoins veiller à adapter le temps d'accès aux parcelles et le chargement en fonction des conditions climatiques et des types de sol afin d'éviter le piétinement excessif des parcelles et de limiter les risques de lessivage des nitrates en automne.

La saison de pâturage peut également être prolongée grâce à la réalisation de stocks sur pied utilisés durant les périodes de croissance faibles à nulles. Des parcelles ne sont plus pâturées en milieu de printemps durant 40 à 60 jours afin de permettre une accumulation d'herbe qui sera ensuite valorisée en été. Cette technique nécessite l'utilisation de prairies d'association avec du trèfle blanc afin de maintenir une valeur nutritive de l'herbe satisfaisante pour alimenter les vaches laitières. Après 70 jours de repousse, la valeur énergétique des prairies de RGATB reste en moyenne proche de 0,75 UFL/kg MS (Delaby et Peccatte 2003) ce qui est similaire à celle d'un bon ensilage d'herbe. Compte tenu des hauteurs d'herbe élevées sur ces parcelles, il est recommandé de les pâturer avec un fil avant (pâturage rationné) afin de limiter le gaspillage.

\section{3 / Le chargement et le système de pâturage ont des effets limités sur les performances}

A partir d'une synthèse bibliographique comportant 36 réponses de production laitière, Delaby et Peyraud (non publié - figure 5) ont montré que l'accroissement d'une unité de chargement moyen annuel (soit une vache en plus par hectare pendant toute la saison de pâturage) altérait les performances individuelles de $1 \mathrm{~kg}$ par vache et par jour (soit $7 \%$ ).

A l'échelle de l'exploitation, la réduction du chargement en vue d'obtenir un accroissement des quantités d'herbe offertes favorables à l'ingestion et à la production laitière n'aura donc finalement qu'un faible impact

Figure 5. Variation de la production laitière individuelle journalière sous l'effet d'un accroissement du chargement par hectare.

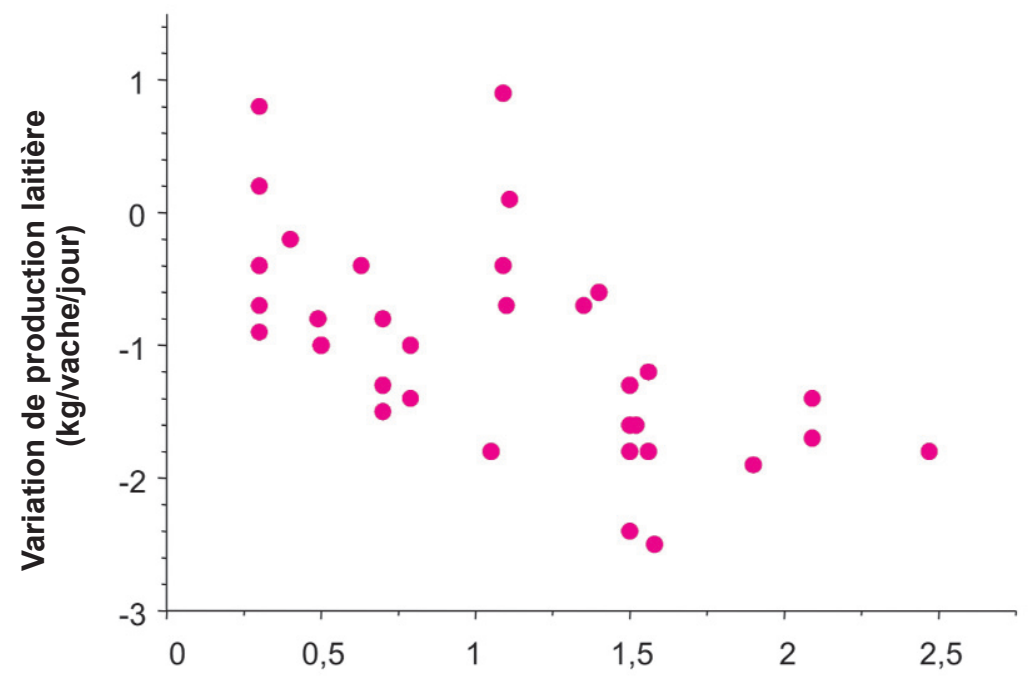

Accroissement du chargement (vache en plus/ha)

sur les performances individuelles. A l'échelle de la parcelle, le chargement affecte directement la hauteur après pâturage. Selon plusieurs auteurs (Hoden et al 1991, O'Brien et al 1999, Delaby et Peyraud 2003), une augmentation de la hauteur d'herbe après pâturage de $1 \mathrm{~cm}$ du fait de la réduction du chargement se traduit par une augmentation de la production laitière individuelle de $1 \mathrm{~kg}$ de lait par jour. A condition de rester en dessous de 7-8 cm, ce centimètre en plus laissé par les animaux semble être la valeur maximale acceptable permettant le maintien d'une herbe de qualité aux repousses suivantes sans avoir recours à des stratégies alternatives telles que la fauche des refus ou le passage d'un second troupeau (Delaby et Peyraud 2003).
Pour un chargement donné, les travaux ayant comparés les systèmes de pâturage tournant ou continu n'ont pas mis en évidence la supériorité de l'un ou l'autre des systèmes (Ernst et al 1980, Hoden et al 1987 - figure 6).

Néanmoins, le pâturage continu semble plus sensible aux conditions climatiques séchantes avec notamment un risque plus important de baisse de la croissance estivale plus conséquente qu'en pâturage tournant (Hoden et al 1987). Ceci confère un certain avantage au pâturage tournant dans les régions où la pluviométrie et/ou la température limitent la croissance de l'herbe. La production laitière individuelle ou par hectare ne diffère pas entre le système de pâturage rationné et le pâturage tour-

Figure 6. Comparaison des productions laitières par hectare obtenues en pâturage tournant ou en pâturage continu (d'après Béranger et Micol 1981).

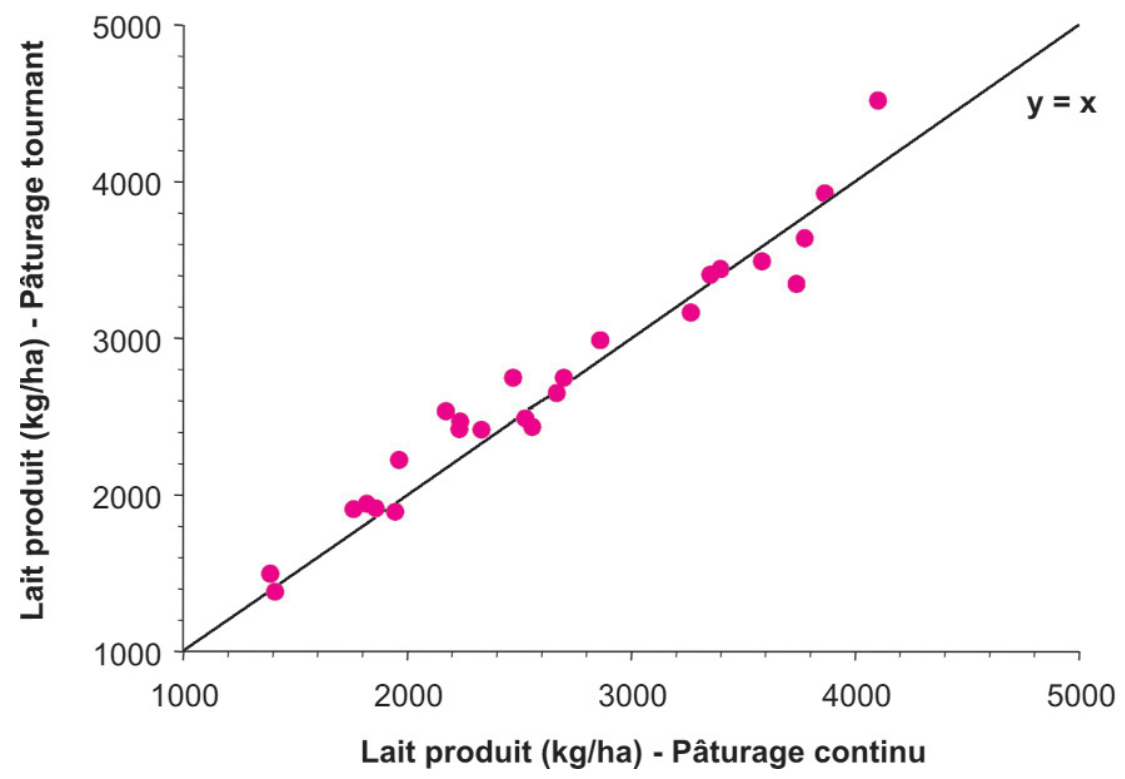


nant simplifié (Hoden et al 1987). La technique de séparation du troupeau en deux lots d'animaux (leader-follower) en fonction de leur niveau de production laitière à la mise à l'herbe a pour objectif d'adapter la sévérité du pâturage aux besoins des animaux. Cependant, le gain de production obtenu chez les animaux du groupe «leader» est très souvent compensé par une perte similaire chez les animaux du groupe «follower» (Mayne et al 1988). La mise en oeuvre d'un tel système assez complexe à gérer au quotidien ne peut se justifier par les gains de productivité qui globalement resteront marginaux. Il peut par contre être intéressant à utiliser dans les exploitations où les fins de parcelles peuvent être valorisées par des génisses ou des boeufs.

\section{4 / Améliorer les pratiques de pâturage et d'alimentation pour améliorer l'utilisation de l'herbe}

Une gestion rigoureuse du pâturage en début de printemps peut avoir des effets bénéfiques sur la qualité de l'herbe jusqu'en début d'été sans affecter la productivité de la prairie. En 2005, O'Donovan et Delaby ont comparé l'effet d'un pâturage précoce en mars (à raison de $6 \mathrm{~h} /$ jour) ou l'absence de pâturage avant mi-avril sur la production laitière et la valorisation de la prairie au cours du printemps. La hauteur en fin de parcelle plus élevée et le taux d'utilisation de l'herbe plus faible observés sur les prairies non pâturées en mars conforte l'idée qu'un pâturage trop tardif par rapport au démarrage de végétation entraîne une accumulation importante de biomasse qui sera difficile à valoriser et qui complique la gestion du pâturage lors des cycles suivants. Dans cet essai, le pâturage précoce a permis une production laitière plus élevée, à condition toutefois d'augmenter un peu la surface offerte (tableau 2), soit une réduction du chargement de 4,6 à 4,0 vaches par hectare. Dans ces conditions, le nombre total de journées de pâturage réalisées par hectare est égal voire supérieur à ceux obtenus avec un premier pâturage plus tardif.

La date de mise à l'herbe a des répercussions très importantes sur la suite de la saison de pâturage. Le risque d'une mise à l'herbe trop précoce est une réalité lorsque la surface accessible aux vaches est faible et/ou lorsque la croissance de l'herbe est limitée lors de printemps froids. Dans ces situations, il devient impossible d'avoir assez d'herbe pour nourrir le troupeau. A partir de simulations, Delaby et Le Gall (2001) ont montré qu'avec 25 ares par vache, soit un chargement de 4 vaches/ha, une mise à l'herbe précoce ( $1^{\mathrm{er}}$ mars) réduit finalement le nombre de jours de pâturage possibles (- 19 jours) et accroît la consommation de fourrages conservés (+85 kg/vache) par rapport à une mise à l'herbe plus tardive (1 ${ }^{\mathrm{er}}$ avril) accompagnée d'une transition plus rapide. A l'inverse, une mise à l'herbe trop tardive induit des situations d'excédents d'herbe et des difficultés à bien valoriser au pâturage une herbe offerte trop haute.

L'intérêt de la complémentation en concentré au pâturage a fait l'objet de revues bibliographiques récentes (Peyraud et Delaby 2001, Delaby et al 2003). Une efficacité de $1 \mathrm{~kg}$ de lait par $\mathrm{kg}$ de MS de concentré consommé est désormais fréquente pour des doses maximales de concentré n'excédant pas 6 kg/vache/jour. Ainsi, l'apport de concentré peut être intéressant pour contraindre les vaches à pâturer plus ras et obtenir une hauteur en sortie de parcelles faible tout en maintenant des performances individuelles convenables à

Tableau 2. Effet de la date du 1er pâturage et du chargement ultérieur sur la production de lait et l'utilisation de l'herbe en mai et juin (O'Donovan et Delaby 2005).

\begin{tabular}{|c|c|c|c|c|}
\hline Date du $1^{\text {er }}$ pâturage & Préco & mars) & Tard & avril) \\
\hline Chargement (vaches/ha) & 4,6 & 4 & 4,6 & 4 \\
\hline Croissance de l'herbe (kg MS /ha/jour) & 66 & 67 & 65 & 58 \\
\hline Herbe offerte (kg MS /vache/jour) (1) & 34,6 & 42,3 & 40,1 & 48,5 \\
\hline Digestibilité de la MO & 0,80 & 0,80 & 0,76 & 0,76 \\
\hline Lait (kg/vache/jour) & 19,6 & 23,1 & 22 & 23 \\
\hline Utilisation de l'herbe (\% des QHO à $5 \mathrm{~cm}$ ) & 104 & 100 & 85 & 81 \\
\hline Hauteur d'herbe après pâturage $(\mathrm{cm})$ & 4,6 & 5 & 6,5 & 6,8 \\
\hline Nbre de jours de pâturage depuis mars (/ha) & 427 & 366 & 350 & 284 \\
\hline
\end{tabular}

(1) Quantités d'Herbe Offertes (QHO) estimées au ras du sol.

un faible coût alimentaire et en assurant la qualité de l'herbe tout au long de la saison de pâturage.

Les éleveurs maintiennent souvent un apport de fourrages conservés afin d'atténuer les variations de production laitière observées au pâturage. En fait, lorsque les conditions de pâturage ne sont pas trop sévères, l'apport de fourrages (ensilage d'herbe, de maïs ou foin) se traduit par un taux de substitution élevé, souvent supérieur à 1 , et par une réponse de production de lait faible ou négative (Leaver 1985, Peyraud et Delaby 2001, Chenais et al 2001) puisque l'on substitue à l'herbe un fourrage de moindre qualité. Ces pratiques contribuent en outre à une mauvaise valorisation de l'herbe. A l'inverse, lors des périodes de pénuries fourragères, l'apport de fourrages se justifie et comporte deux objectifs. Il permet d'une part de maintenir les niveaux d'ingestion et de production des vaches puisque le taux de substitution est alors faible (moins de 0,3) et d'autre part d'accroître la durée des cycles de pâturage et d'accumuler de l'herbe pour les périodes de pâturage suivantes.

\section{3 / Méthodes et outils dispo- nibles pour donner confian- ce aux éleveurs herbagers}

La gestion du pâturage consiste à trouver l'optimum entre deux objectifs : de bonnes performances animales et une bonne valorisation de l'herbe produite.

\section{1 / Alimentation à l'échelle de la parcelle en cours de pâturage}

La hauteur de l'herbe après pâturage est un critère descriptif de l'état du couvert végétal qui peut être utilisé à deux fins pour la gestion du pâturage. En terme d'alimentation de l'animal, une hauteur faible en fin de parcelle traduit des apports nutritifs limitants et des quantités ingérées inférieures à l'ingestion possible. Une hauteur élevée traduit à l'inverse une sous utilisation de l'herbe dans la parcelle considérée. Comme la hauteur en sortie de parcelle est en partie dépendante de celle observée en entrée de parcelle, l'ingestion d'herbe est mieux prédite lorsque la hauteur de fin de parcelle est exprimée en proportion de la hauteur entrée plutôt qu'en valeur absolue (Delagarde et al 2001a). En terme de gestion du pâturage, des hauteurs élevées indiquent 
une accumulation d'herbe résiduelle qu'il sera difficile à valoriser aux cycles suivants. Ainsi, en Bretagne, pour faciliter les décisions de sortie de parcelle, des hauteurs objectifs ont été proposées par les organismes de développement (Dequin et al 1998, Seuret et al 2004). Ces hauteurs objectifs évoluent de 4,5 à $6 \mathrm{~cm}$ entre le début du printemps et l'été. Une approche similaire a été proposé en Irlande (Stakelum et al 1997) mais avec une seule hauteur de référence pour toute la saison de pâturage $(5,5 \mathrm{~cm})$.

La hauteur en entrée de parcelle peut aussi être utilisée comme critère d'aide à la gestion du pâturage. Une hauteur d'herbe mesurée à l'herbomètre supérieure à $16 \mathrm{~cm}$ correspond à une parcelle qui sera difficile à pâturer et induit une diminution de l'ingestion (Peyraud et al 1996). L'optimum de hauteur se situe entre 12 et $14 \mathrm{~cm}$ en entrée de parcelle sur prairies de RGA ou de RGATB. En Irlande, Stakelum et al (1997) propose comme objectif en entrée de parcelle, une biomasse (au dessus de $4 \mathrm{~cm}$ ) de $2000 \mathrm{~kg}$ de MS, soit environ $12 \mathrm{~cm}$ de hauteur mesurée à l'herbomètre.

Afin de faciliter le rationnement du troupeau à l'échelle de la parcelle, un modèle de prévision des quantités d'herbe ingérées au pâturage (GrazeIN) a été récemment développé (Delagarde et al 2004a) dans le cadre du projet européen «Grazemore» (Mayne et al 2004). GrazeIN permet de prévoir l'ingestion d'un groupe de vaches conduit en pâturage tournant ou continu dans une large gamme de situations concernant les animaux, les prairies, la complémentation et les pratiques de pâturage. GrazeIN prend en compte la capacité d'ingestion des vaches, les quantités d'herbe offertes (figure 2), la biomasse initiale en pâturage tournant ou la hauteur de l'herbe en pâturage continu ainsi que le temps d'accès au pâturage dans la journée. Ce modèle de prévision de l'ingestion va être intégré au logiciel INRAtion dont l'utilisation pourra alors être étendue au pâturage.

\section{2 / Alimentation à plus long terme à l'échelle de l'exploita- tion}

Le chargement a été le premier critère proposé pour gérer le pâturage et les surfaces pâturées. Un chargement élevé est recommandé au printemps suivi d'une diminution adaptée à la région en été-automne. Comme cette notion de chargement reste imprécise et peu spécifique de chaque exploitation, une méthode basée sur des dates-clefs a été proposée afin de gérer plus précisément le pâturage. Elle constitue un premier progrès. Ces dates correspondent à des moments particuliers de la saison de pâturage où l'éleveur doit prendre des décisions importantes. Au printemps, il s'agit des dates de mise à l'herbe, de pâturage «jour et nuit», de fermeture du silo. Durant la saison de pâturage, ces dates concernent la réouverture du silo, le retour en stabulation la nuit ou la fin de la saison de pâturage. En Bretagne, 6 stratégies de gestion du pâturage pour les vaches laitières (Seuret et al 2004) ont été proposées par les ingénieurs de développement spécialistes du pâturage. Ces stratégies ont été adaptées au contexte local pour tenir compte de la surface accessible (de 23 à 70 ares), de la zone pédoclimatique et de la durée de la saison de pâturage seul (de 0 à 210 jours).

Le concept de trésorerie fourragère proposé par Duru et al (1988) permet d'évaluer les variations et l'évolution de la quantité d'herbe accumulée au cours du temps. Le stock d'herbe disponible (SHD en kg MS) correspond à la biomasse (au dessus de $5 \mathrm{~cm}$ ) présente un jour donné sur l'ensemble des parcelles. Cette valeur peut ensuite être exprimée en jours d'avance en divisant le SHD par le nombre de vaches du troupeau et une évaluation des quantités d'herbe ingérées (par exemple, $15 \mathrm{~kg} /$ vache/jour; Chenais et al 2001). Les recommandations en matière de jours d'avance varient en fonction de la surface accessible par vache et de la saison. Elles évoluent de 8-10 jours à la mise à l'herbe (mars-avril) à 1015 jours au printemps pour atteindre la valeur de 25 à 50 jours en été selon la croissance estivale escomptée (Seuret et al 2004). Une approche similaire a été développée en Irlande mais le stock d'herbe disponible, appelé «Farm Cover», est exprimé en $\mathrm{kg}$ de $\mathrm{MS} / \mathrm{ha}$ au dessus de $4 \mathrm{~cm}$. L'évaluation de la biomasse présente sur chaque parcelle est réalisée chaque semaine par l'éleveur, soit par estimation visuelle, soit grâce à la mesure de hauteurs réalisée à l'herbomètre en parcourant l'ensemble des parcelles et à une estimation de la densité exprimée en $\mathrm{kg}$ de MS par $\mathrm{cm}$ et par hectare. Une étude récente (Defrance et al 2004), réalisée à partir de données issues des fermes expérimentales de l'Ouest de la France (Bretagne, Normandie) a permis de préciser les variations mensuelles de cette densité de l'herbe.
Les méthodes et outils décrits précédemment présentent le défaut de rester statiques et de ne pas prendre en compte toute la complexité des interactions entre les décisions du moment et les réactions en chaîne induites à plus long terme sur la croissance de l'herbe et l'ingestion. Des modèles (Pâtur'IN : Delaby et al 2000, Sepatou : Cros et al 2003, GrazeMore : Mayne et al 2004) ont été développés afin de simuler, sur plusieurs mois et dans différents contextes de climat, les conséquences des différentes stratégies de gestion de l'alimentation et du pâturage. Tous ces modèles intègrent un sous-modèle «Plante» pour simuler la croissance et l'accumulation de biomasse, un sousmodèle «Troupeau» pour simuler l'ingestion et la production laitière (GrazeMore) et un sous-modèle «Décision» afin de reproduire les différentes stratégies (système de pâturage, chargement, fertilisation, fauche, complémentation). Les sous-modèles «Plante» et «Animal» sont toujours construits en vue d'intégrer les interactions entre ces deux composantes des systèmes pâturés. Par contre, les fonctionnalités et interfaces différent selon les outils. A l'heure actuelle, ces outils permettent de réfléchir à des stratégies de conduite mais ils ne sont pas très pertinents et/ou trop complexes pour une utilisation tactique en exploitation. Ils sont, pour certains, des outils de recherches sur les systèmes fourragers et fournissent un cadre rigoureux pour confronter différentes stratégies. Ces modèles peuvent aussi être utilisés dans le cadre de l'enseignement sur le pâturage afin de permettre aux élèves de mieux comprendre l'ensemble des processus qui contrôlent les systèmes laitiers herbagers. Une première expérience a été mise en place en France en 2002 en relation avec le CNERTA (Delaby et al 2002). Dans le cadre du GIS Agrotransfert Bretagne, ces modèles servent actuellement à développer des outils d'aide à la gestion du pâturage simples, efficaces et peu exigeants en temps en vue d'une utilisation en exploitation (Defrance et al 2005).

\section{Conclusion}

Quels futurs challenges pour les recherches sur le pâturage afin de maximiser à la fois la valorisation de l'herbe et les performances animales ?

Les connaissances acquises ces dernières années concernant l'ingestion au pâturage et la croissance de l'herbe of- 
frent des perspectives encourageantes en matière de gestion du pâturage et des systèmes herbagers. Il reste néanmoins d'autres défis à relever dans les 10 prochaines années. i) Le premier d'entre eux restera la maximisation de l'ingestion d'herbe. Concernant la régulation de l'ingestion d'herbe, il semble que les travaux autour des relations entre structure de l'herbe et taille des bouchées atteignent leurs limites. Il paraît nécessaire de développer des travaux de recherches sur l'analyse des contraintes physiques et/ou métaboliques de l'ingestion afin de mieux comprendre pourquoi les quantités ingérées d'herbe sont plus faibles que celles de rations à base d'ensilage de maïs malgré une digestibilité plus élevée. On peut soupçonner des freins liés au trafic des particules dans le rumen (notamment le temps de réduction de taille de ces particules), à la teneur en eau élevée de l'herbe. ii) Le second challenge concernera le maintien d'une proportion de feuilles vertes importante dans la structure du couvert végétal afin de maintenir un niveau d'ingestion élevé tout en obtenant une hauteur en sortie de parcelle faible qui facilite la gestion du pâturage. Il s'agit ici de mieux comprendre comment les arrières effets favorables d'un pâturage précoce au printemps peuvent persister durant la saison de pâturage et comment un pâturage précoce affecte les performances par hectare lors des cycles suivants. iii) La troisième source de progrès possible concernera la gestion et la prévision de croissance de l'herbe et l'amélioration de la disponibilité de l'herbe à l'échelle de l'exploitation. L'objectif est principalement d'allonger la saison de pâturage tôt au printemps, plus tard en automne ou durant l'été alors que la croissance est limitée par les conditions climatiques. De même, dans les régions favorables à la culture du maïs destiné à l'ensilage, il peut être intéressant de limiter les excédents d'herbe au printemps afin de limiter la récolte par fauche qui est coûteuse et conduit à des fourrages conservés de moindre valeur alimentaire que l'ensilage de maïs. Dans ces régions, l'herbe doit d'abord être valorisée par le pâturage. Il serait intéressant de mobiliser la sélection variétale dans l'objectif de modifier la saisonnalité de la croissance de l'herbe en l'associant avec les pratiques de fertilisation azoté et de pâturage. Dans cette situation, le trèfle blanc présente un réel intérêt puisqu'il pousse moins vite que les graminées au printemps et surtout permet des âges de repousse élevés (50-60 jours) sans pénalisation excessive de sa valeur nutritive. iv) Le quatrième champ d'investigations devra s'intéresser aux méthodes et outils d'aide à la décision qui encourageront les éleveurs à utiliser le pâturage en améliorant leur confiance dans les systèmes herbagers. Pour être opérationnels en exploitation, ces méthodes et outils doivent être simples d'utilisation, nécessiter un temps d'enregistrement limité et des variables d'entrée facilement disponibles. Les modèles de gestion du pâturage servent principalement aux chercheurs et ingénieurs afin d'affiner les stratégies de gestion du pâturage et de réfléchir à d'autres itinéraires techniques. Ces outils d'aide à la réflexion permettent de simuler au moindre coût de nom-breux scénarios afin de mieux quantifier les risques et conséquences de tel ou tel choix technique et d'aider aux échanges de point de vue. Finalement, un renforcement des collaborations entre la recherche et les organismes de développement agricole s'avère nécessaire pour la mise au point d'outils conviviaux réellement destinés à la prise de décision au quotidien en exploitation agricole.

\section{Références}

Béranger C., Micol D., 1981. Utilisation de l'herbe par les bovins au pâturage. Importance $\mathrm{du}$ chargement et du mode d'exploitation. Fourrages, 85, 73-93.

Bourgeois L., 2002. Common agricultural policy and grasslands: the case study of France. Grassland Science in Europe, 7, 5-15.

Buckley F., Dillon P., Rath M., Veerkamp R.F., 2000. The relationship between genetic merit for yield and live weight condition score, and energy balance of spring calving Holstein Friesian dairy cows on grass based systems on milk production. J. Dairy Sci., 83, 1878-1886.

Chatellier V., Vérité R., 2003. L'élevage bovin et l'environnement en France : le diagnostic justifie-t-il des alternatives techniques ? INRA Prod. Anim., 16(4), 231-249.

Chausson F., 2004. La réforme de la politique laitière européenne : des accords de Luxembourg à l'accord interprofessionnel sur le prix du lait. Acte du séminaire de l'AFPF, Paris, France, 28 Oct. 2004, 25-32.

Chenais F., Le Roux M., 1996. Réduction de la part de maïs dans les systèmes d'alimentation des vaches laitières. Résultats expérimentaux obtenus en Bretagne. Document EDE-Chambre d'Agriculture de Bretagne, 12-15.

Chenais F., Seuret J.M., Brunschwig P., Fiorelli J.L., 2001. Pour un rôle croissant du pâturage dans les systèmes bovins laitiers. Fourrages, 166, 257-277.
Cros M.J., Duru M., Garcia F., MartinClouaire R., 2003. A biophysical dairy farm model to evaluate rotational grazing management strategies. Agronomie, 23, 105-122.

Defrance P., Delaby L., Seuret J.M., 2004. Mieux connaître la densité de l'herbe pour calculer la croissance, la biomasse d'une parcelle et le stock d'herbe disponible d'une exploitation. Renc. Rech. Rum., 11, 291-294.

Defrance P., Seuret J.M., Delaby L., 2005. Grass growth profiles in Brittany. In: Utilisation of grazed grass in temperate animal systems. J.J. Murphy (Ed.). Proc. 20 th Int. Grassland Congr., Cork Satellite Workshop, 3-6 July, Ireland, 214.

Defrance P., Delaby L., Seuret J.M., 2005. Herb'Avenir : un outil simple d'aide à la décision pour la gestion du pâturage, Renc. Rech. Rum., 12, sous presse.

Delaby L., Le Gall A., 2001. Influence de la surface en herbe disponible et de la date de mise à l'herbe sur les principales caractéristiques du pâturage des vaches laitières au printemps. Renc. Rech. Rum., 8, 223.

Delaby L., Peccatte J.R., 2003. Valeur alimentaire des prairies d'association ray-grass anglais/trèfle blanc utilisées entre 6 et 12 semaines d'âge de repousse. Renc. Rech. Rum., 10, 389.

Delaby L., Peyraud J.L., 2003. The effect of two contrasting grazing managements and level of concentrate supplementation on the performance of grazing dairy cows. Anim. Res., 52, $437-460$
Delaby L., Peyraud J.L., Faverdin P., 2000. Développement d'un organiseur de pâturage assisté par ordinateur : Pâtur'IN. Renc. Rech. Rum., 7, 329-332.

Delaby L., Peyraud J.L., Delagarde R., 2001. Effect of the level of concentrate supplementation, herbage allowance and milk yield at turnout on the performance of dairy cows in mid lactation at grazing. Anim. Sci., 73, 171-181.

Delaby L., Peyraud J.L., Delagarde R., 2003. Faut-il complémenter les vaches laitières au pâturage ? INRA Prod. Anim., 16(3), 183-195.

Delaby L., Fontirroig S., Granger S., Pierret P., Pillet J.M., 2002. Pâtur'IN expliqué aux élèves. CNERTA (eds). Livret pédagogique, $95 \mathrm{p}$.

Delagarde R., Faverdin P., Baratte C., Peyraud J.L., 2004a. Prévoir l'ingestion d'herbe et la production des vaches laitières: GrazeIN, un modèle pour raisonner l'alimentation au pâturage. Renc. Rech. Rum., 11, 295-298.

Delagarde R., Peyraud J.L., Parga J., Ribeiro H., 2001a. Caractéristiques de la prairie avant et après un pâturage : quels indicateurs de l'ingestion chez la vache laitière. Renc. Rech. Rum., 8 , 209-212.

Delagarde R., Peyraud J.L., Delaby L., $2004 b$. High grazing pressure in early-spring increases herbage intake of grazing dairy cows in latespring. In: Land Use Systems in Grassland Dominated Regions. Proc. $20^{\text {th }}$ General Meeting of EGF, 21-24 June, Luzern, Switzerland, 647649. 
Delagarde R., Prache S., D'Hour P., Petit M., 2001b. Ingestion de l'herbe par les ruminants au pâturage. Fourrages, 166, 189-212.

Dequin A., Follet D., Grasset M., Le Gall A., Roger P., Thebault M., 1998. Cinq menus pour vaches laitières au pâturage. Document EDEChambres d'Agriculture de Bretagne. 12p. + fiches.

Dillon P., Crosse S., O’Brien B., Mayes R.W., 2002. The effect of forage type and level of concentrate supplementation on the performance of spring-calving dairy cows in early lactation. Grass and Forage Sci., 57, 212-223.

Duru M., Fiorelli J.L., Osty P.L., 1988. Proposition pour le choix et la maitrise du système fourrager. I. Notion de trésorerie fourragère. Fourrages, 113, 37-56.

Ernst P., Le Du Y.L.P., Carlier L., 1980. Animal and sward production under rotational and continuous grazing management; a computer simulation of practical and economic implications. Grass and Forage Sci., 38, 261-272.

Gowen N., O'Donovan M., Casey I., Rath M., Delaby L., Stakelum G., 2003. The effects of grass cultivars differing in heading date and ploidy on the performance and dry matter intake of spring calving dairy cows at pasture. Anim. Res., 321-336.

Hageman I.W., Latinga E.A., Schlepers H., Neuteboom J.H., 1993. Herbage intake, digestibility characteristics and milk production of a diploid and two tetraploid cultivars of perennial ryegrass. Proc. $17^{\text {th }}$ Int. Grassland Congr., Palmeston North, USA, 459-460.

Harris S.L., Clark D.A., Auldist M.J., Waugh C.D., Laboyrie P.G., 1997. Optimum white clover content for dairy pastures. Proc. N. Z. Grassland Ass., 59, 29-33.

Hoden A., Fiorelli J.L., Jeannin B., Huguet L., Muller A., Weiss P., 1987. Le pâturage simplifié pour vaches laitières : synthèse de résultats expérimentaux. Fourrages, 111, 239-257.

Hoden A, Muller A., Peyraud J.L., Delaby L., Faverdin P., 1991. Pâturage pour vaches laitières. Effets du chargement et de la complémentation en pâturage tournant simplifié. INRA Prod. Anim, 4(3), 229-239.

Holmes W., 1980. Grazing management. In: Grass: its production and utilization. W. Holmes (Ed) BGS Blakwell, Owford, 125-173.

Hoogendoorn C.J., Holmes C.W., Chu A.C.P., 1992. Some effects of herbage composition as influenced by previous grazing management, on milk production by cows grazing on ryegrass / white clover pastures. 2. Milk production in late spring / summer: effect of grazing intensity during the preceding spring period. Grass and Forage Sci., 47, 316-325.

Kennedy J., Dillon P., Delaby L., Faverdin P., Stakelum G., Rath M., 2002a. Effect of genetic merit and concentrate supplementation on grass intake and milk production with Holstein friesian dairy cows. J. Dairy Sci., 86, 610-621.

Kennedy J., Dillon P., Faverdin P., Delaby L., Buckley F., Rath M., 2002b. The influence of cow genetic merit for milk production on response to level of concentrate supplementation in a grass-based system. Anim. Sci., 75, 433-445.

Leaver J.D., 1985. Milk production from grazed temperate grassland. J. Dairy Res., 52, 313344.
Le Gall A., Raison C., Bertrand S., Dockès A.C., Pflimlin A., 2004. Impacts de la conditionnalité environnementale des aides de la Politique Agricole Commune sur les systèmes laitiers français. Acte du séminaire de l'AFPF, Paris, France, 28 Oct. 2004, 45-63.

Lemaire G., Salette J., 1981. Prévision des potentiels de production en fonction des conditions pédoclimatiques : application au pâturage. Fourrages, 85, 23-37.

Mayne C.S., Laidlaw A.S., 1995. Extending the grazing season, a research review. In: Extending the Grazing Season, Discussion Meeting, Proc. British Grassland Soc., Reaseheath College, Nanwich, Cheshire, 6-11.

Mayne C.S., Newberry R.D., Woodcock S.C.F., 1988. The effects of a flexible grazing management strategy and leader / follower grazing on the milk production of grazing dairy cows and sward characteristics. Grass and Forage Sci., 43, 137-150.

Mayne C.S., Newberry R.D., Woodcock S.C.F., Wilkins R.J., 1987. Effect of grazing severity on grass utilization and milk production of rotationally grazed dairy cows. Grass and Forage Sci., 42, 59-72.

Mayne C.S., Rook A.J., Peyraud J.L., Cone J., Martinsson K., Gonzales A., 2004. Improving sustainability of milk production systems in Europe through increasing reliance on grazed pasture. In: Land Use Systems in Grassland Dominated Regions. Proc. $20^{\text {th }}$ General Meeting of EGF, 21-24 June, Luzern, Switzerland, 584586.

Mc Gilloway D.A., Mayne C.S., 1996. The importance of grass availability for the high genetic merit cow. In: P.C. Garnsworthy, J. Wiseman, W. Haresign (Eds), Recent Advances in Animal Nutrition. Nottingham University Pres, Nottingham, UK., 135-169.

Mosquera-Losada MR., Gonzalez-Rodriguez A., 1999a. Pasture production in Northen Spain dairy systems. N. Z. J. Agric. Res., 42, 125-132.

Mosquera-Losada MR., Gonzalez-Rodriguez A., 1999b. Use of first nitrogen in South Europe temperate grassland. $10^{\text {th }}$ Nitrogen Workshop, 232-233.

O’Brien B., Dillon P., Murphy J.J., Mehra R.J., Guinee T.P., Connolly J.F., Kelly A., Joyce P., 1999. Effect of stocking density and concentrate supplementation of grazing dairy cows on milk production, composition and processing characteristics. J. Dairy Res., 66, 165-176.

O'Donovan M., Delaby L., Stakelum G., Dillon P., 2004. Effect of autumn/spring nitrogen application date and level on dry matter production and nitrogen efficiency in perennial ryegrass swards. Irish J. Agric. Fd Res., 43 (1), 31-42.

O’Donovan M., Delaby L., Peyraud J.L., 2005. Effect of time of initial grazing date and subsequent stocking rate on pasture production and dairy cow performance. Anim. Res., 53, 1-11.

Parga J., Peyraud J.L., Delagarde R., 2000. Effect of sward structure and herbage allowance on herbage intake by grazing dairy cows. In: A.J. Rook, P.D. Penning (Eds), Grazing management: The principles and practice of grazing for profit and environmental gain in temperate grassland system. BGS Occasional Symposium, 34, 61-66.
Parris K., 2002. Grasslands and the environment: recent European trends and future direction - an OECD perspective. In: Multi-function grasslands. Proc. of the $19^{\text {th }}$ General Meeting of EGF, 25-30 May, La Rochelle, France, 957-985.

Peyraud J.L., Comeron E.A., Wade M., Lemaire G., 1996. The effect of daily herbage allowance, herbage mass and animal factors upon herbage intake by grazing dairy cows. Ann. Zootech., 45, 201-217.

Peyraud J.L., Delaby L., 2001. Ideal concentrate feeds for grazing dairy cows - Response to concentrates in interaction with grazing management and grass quality. In: P.G. Garnsworthy, J. Wiseman (eds), Recent Advances in Animal Nutrition. University of Nottingham University Press, UK., 203-220.

Ribeiro-Filho H.M.N., Delagarde R., Peyraud J.L., 2003. Inclusion of white clover in stripgrazed perennial ryegrass swards: herbage intake and milk yield of dairy cows at different ages of sward regrowth. Anim. Sci., 77, 499-510.

Sayers H.J., Mayne C.S., 2001. Effect of early turnout to grass in spring on dairy cows performance. Grass and Forage Sci., 56, 259-267.

Seuret J.M., Jégou V., Portier B., Grasset M., 2004. Optimiser l'alimentation et la conduite. Guide pratique de l'éleveur laitier, Chambres d'agriculture-EDE de Bretagne, 67p.

Stakelum G., Connolly J., 1987. Effect of body size and milk yield on intake of fresh herbage by lactating dairy cows indoors. Irish J. Agric. Res., 26, 9-22.

Stakelum G., O'Donnovan M., Maher J., 1997. Simplified grazing management for high performances from the cows. In: Technology for profitable farming. National Dairy Conference, Fermoy $20^{\text {th }}$ March, Teagasc, 33-53.

Thomson D.J., Beever D.E., Haines M.J., Cammell S.B., Evans R.T., Dhanoa M.S., Austin A.R., 1985. Yield and composition of milk from friesian cows grazing either perennial ryegrass or white clover in early lactation. J. Dairy Res., $52,17-31$.

Wade M.H., Peyraud J.L., Lemaire G., Comeron E.A., 1989. The dynamic of daily area and depth of grazing and herbage intake of cows in a five day paddock system. $16^{\text {th }}$ Int. Grassland Congr., Nice, France, 1111-1112.

Wade M.H., Peyraud J.L., Comerón E.A., Lemaire G., 1995. The dynamics of daily milk production and sward height under paddock grazing conditions. Ann. Zootech., 44, Suppl., 127.

Wilkins R.J., Gibb M.J., Huckle C.A., 1995. Lactation performance of spring-calving dairy cows grazing mixed perennial ryegrass / white clover swards of differing composition and height. Grass and Forage Sci., 50, 199-208.

Wilkins R.J., Gibb M.J., Huckle C.A., Clements A.J., 1994. Effect of supplementation on production by pring-calving dairy cows grazing swards of differing clover content. Grass and Forage Sci., 49, 465-75.

Wilson D., 1993. Breeding grasses and legumes for an extended grazing season. In: The place for grass in land use systems. British Grassland Society, December 1993, 93-104. 


\title{
Résumé
}

Les prairies et le pâturage devraient constituer à l'avenir la base des systèmes laitiers durables. L'herbe pâturée est le fourrage le moins coûteux pour alimenter les vaches laitières et constitue un mode d'alimentation qui réduit l'impact environnemental des systèmes très intensifs tout en conférant une image plus «naturelle» aux systèmes laitiers. L'utilisation efficace de l'herbe au pâturage nécessite la mise au point de méthodes qui permettent de maximiser l'ingestion journalière d'herbe tout en assurant la production d'une quantité importante d'une herbe de qualité durant toute la saison de pâturage. Les opportunités d'accroître la part de l'herbe pâturée dans l'alimentation des vaches laitières existent en allongeant la saison de pâturage au printemps et/ou en automne et en pratiquant le report d'herbe sur pied en été. A ce titre, l'introduction de légumineuses, trèfle blanc notamment, dans les prairies doit être favorisée. Les possibilités de moduler la saisonnalité de la croissance de l'herbe existent au travers de la sélection fourragère, de la stratégie de fertilisation azotée ou des pratiques de pâturage. L'intérêt d'une réduction du chargement visant à maximiser les performances individuelles reste limité compte tenu des effets cumulatifs défavorables sur la qualité de l'herbe et sa valorisation. Par contre, l'accroissement de la proportion de feuilles grâce à des pratiques de pâturage appropriées au printemps permet d'accroître l'ingestion et de maintenir une hauteur résiduelle faible qui facilite la gestion du pâturage. Il reste à en évaluer les conséquences durant toute la saison de pâturage et son influence sur les performances annuelles par hectare. Dans un futur proche, le développement de méthodes et d'outils d'aide à la gestion du pâturage à la fois pour le rationnement des vaches laitières et pour la gestion des parcelles devrait donner confiance aux éleveurs et favoriser le retour du pâturage dans l'alimentation des vaches laitières.

\begin{abstract}
Combining the optimal management of grazing and the performances of dairy cows: issue and tools

Utilisation of grazed grass should form the basis of sustainable dairy systems in the future. Grazed grass is the cheapest feed available for dairy cows and may also help to reduce the environmental impact of the most intensive systems while giving a natural image of farming. Efficient exploitation of grass by grazing will require the development of grazing systems designed to maximise daily herbage intake per cow while maintaining a greater quantity of high quality pasture over the grazing season. This paper discusses the conditions for efficient exploitation of grass and outlines the possibilities for future advances. There are opportunities to consume more grass by extending the grazing season in early spring and late autumn or by intentionally deferring grazing from a period of grass surplus in the spring to a period of grass shortage in the summer. The introduction of legumes in grass-based swards should also be seriously considered as an alternative option to pure grass swards in order to maximise cow performance. There are also opportunities to manage seasonal production of grass by plant selection, improved $\mathrm{N}$ fertilisation strategies and better grazing management. The opportunity to increase cow performance by decreasing stocking rates is rather limited because this practise is constrained by the necessity to maintain high quality swards over the grazing season, unless alternative strategies are adopted. Increasing leaf blade mass at the base of the sward by appropriate grazing management in the early spring may play a major role in increasing herbage intake. This will result in a low residual sward height thus making grazing management easier. This requires a greater knowledge of the carryover effects of early season grazing management on mid-season pasture quality and the implication for milk output per hectare. In the future, development of reliable decision support methods and tools for short term rationing of grass at the paddock level and long term budgeting of grass at the farm level will give farmers more confidence in grazing and will undoubtedly contribute to optimising the proportion of grazed grass in the annual diet of the dairy cow.
\end{abstract}

PEYRAUD J.-L., DELABY L., 2005. Combiner la gestion optimale du pâturage et les performances des vaches laitières : enjeux et outils. INRA Prod. Anim., 18, 231-240. 